\title{
Pharmacotherapy of treatment-resistant schizophrenia: a clinical perspective
}

\author{
Markus Dold, ${ }^{1}$ Stefan Leucht ${ }^{1,2}$
}

${ }^{1}$ Department of Psychiatry and Psychotherapy, Technical University Munich, Klinikum rechts der Isar, Munich, Germany; ${ }^{2}$ Department of Psychiatry, University of Oxford, Oxford, UK

\begin{abstract}
A significant number of patients with schizophrenia do not respond adequately to an initial antipsychotic trial. As first step within a treatment algorithm for therapy-refractory schizophrenia 'pseudoresistance' should be ruled out (eg, re-evaluation of the diagnosis, comorbidities, compliance and adherence in terms of medication intake, adequate dose and treatment duration, and achievement of sufficient plasma levels). In case of treatment resistance, two strategies that are often used in clinical routine care contain dose increase of the current administered antipsychotic drug (dose escalation, high-dose treatment) and switch to another, new antipsychotic. Although the response rates for both options are generally rather low, we see from the evidence-based perspective a slight advantage of the switching strategy (preferably to an antipsychotic with a different receptor-binding profile) compared to a high-dose treatment. After treatment failures with at least two different antipsychotic drugs, a monotherapy with clozapine is considered to be the treatment option of first choice. At present, pharmacological combination and augmentation strategies cannot be regarded as a generally recommendable evidence-based treatment method. Antipsychotic monotherapy should be preferably sought. In case of combination treatment, it appears more appropriate to combine preferentially two antipsychotics with different receptor-binding profiles.

Augmentation of antipsychotics with other agents should be used primarily to treat specific target symptoms.
\end{abstract}

Treatment resistance is one of the most important clinical challenges in the pharmacological management of schizophrenia. Although precise estimates are missing, authors assume that approximately $20-30 \%$ of all patients with schizophrenia do not respond adequately to an initial antipsychotic trial. ${ }^{1}$ Therefore, the evaluation of therapeutic options in case of treatment resistance is highly clinically relevant. In this article we summarise the evidence for pharmacological treatment options in therapy-refractory schizophrenia derived from clinical trials, systematic reviews, meta-analyses and treatment guidelines. At present, a uniform definition of treatment resistance in the pharmacotherapy of schizophrenia is not available. ${ }^{2}$ Most treatment guidelines require the failure of at least two antipsychotic trials with different compounds, including at least one second-generation antipsychotic, in adequate dose over a period between 2 and 8 weeks before treatment resistance can be assumed. $^{3-5}$ In their review article, Suzuki et $a l^{2}$ evaluated the criteria that were applied in 33 psychopharmacological studies to define treatment resistance. The majority of clinical trials requested non-response to at least two previous antipsychotic trials (chlorpromazine equivalents between 400 and $1000 \mathrm{mg} /$ day) for at least 6 weeks. A significant proportion of the trials applied resistance criteria based on the strict so-called 'Kane criteria' which were introduced in 1988 in the context of a double-blind, randomised trial comparing clozapine with chlorpromazine in treatment-resistant schizophrenia. ${ }^{6}$ In this study (the first to demonstrate the superiority of clozapine in treatment-resistant schizophrenia) patients had to be (1.) non-responsive to at least three periods of treatment in the preceding 5 years with antipsychotic agents (from at least 2 different chemical classes) at dosages equivalent to or greater than $1000 \mathrm{mg} /$ day of chlorpromazine for a period of 6 weeks, each without significant symptomatic relief, and (2.) without any period of good functioning within the preceding 5 years. Moreover, to verify treatment resistance, all participants had to be non-responders to a prospective trial with haloperidol (up to $60 \mathrm{mg} /$ day) that was conducted before randomisation to either clozapine or chlorpromazine.

When discussing the different approaches to define treatment resistance, it should be noted that in the past a lack of improvement primarily in the positive symptoms of schizophrenia was considered. Other schizophrenic symptom domains and functional outcomes such as social functioning or quality of life were not incorporated sufficiently. At present, long-term treatment aims ('recovery') receive more attention resulting in broader definitions of treatment resistance in schizophrenia. ${ }^{7}$
It is interesting to note that a neuroimaging study using $\left[{ }^{18} \mathrm{~F}\right]-\mathrm{DOPA}$ positron emission tomography scanning examined the underlying neurobiological pathophysiology of resistance to antipsychotic medication by comparing non-responsive patients with those who had responded adequately to antipsychotics and healthy volunteers. ${ }^{8}$ In this investigation, the dopamine synthesis capacity was lower in participants with treatment-resistant schizophrenia than in those with sufficient response to antipsychotic medication. Moreover, there was no significant difference between participants with treatment-resistant schizophrenia and healthy volunteers. These findings suggest that a medication aimed to achieve a blockade of dopamine receptors may be effective especially in patients who have an elevated dopamine synthesis capacity but less efficacious in patients with relatively normal levels of dopamine synthesis capacity and in treatment-resistant patients.

The present review is a shorter version of a report on this topic published in German ${ }^{9}$ and also updates previous articles of our group, ${ }^{10} 11$ which had, however, a broader scope and the current one has been entirely rewritten and amended. This report is based on a MEDLINE search with the keyword 'antipsychotic ${ }^{* \prime}$ or 'schizophreni*'. We aimed to give clinicians an overview of the available pharmacological treatment options for treatment-resistant schizophrenia.

\section{Operationalising treatment resistance}

If a patient does not respond adequately to the first administered antipsychotic drug, the following steps should be considered (figure 1):

1. Re-evaluation of the diagnosis of a schizophrenic disorder. Particularly severe personality disorders, mania or depressive disorders with psychotic features are in their acute phase sometimes difficult to distinguish from schizophrenia. Similarly, brain tumours and encephalopathies can cause psychotic states. Furthermore, substance abuse might need to be ruled out. Comorbidities such as affective disorders or obsessive-compulsive disorder should be considered, as these may contribute to treatment resistance.

2. Possible non-compliance of the patient regarding medication intake. Non-compliance or non-adherence can be considered as a major reason for non-response to antipsychotic medication. It has been assumed that more than half of the patients do not take the prescribed medication correctly. ${ }^{12}$ To objectify compliance and adherence in terms of medication intake, plasma levels should be considered. The use of long-acting injectable antipsychotics can sometimes be a possibility to rule out non-adherence. 
Figure 1 Algorithm for the pharmacological treatment of patients with treatment-resistant schizophrenia (modified from refs ${ }^{3}$ and ${ }^{4}$ ).

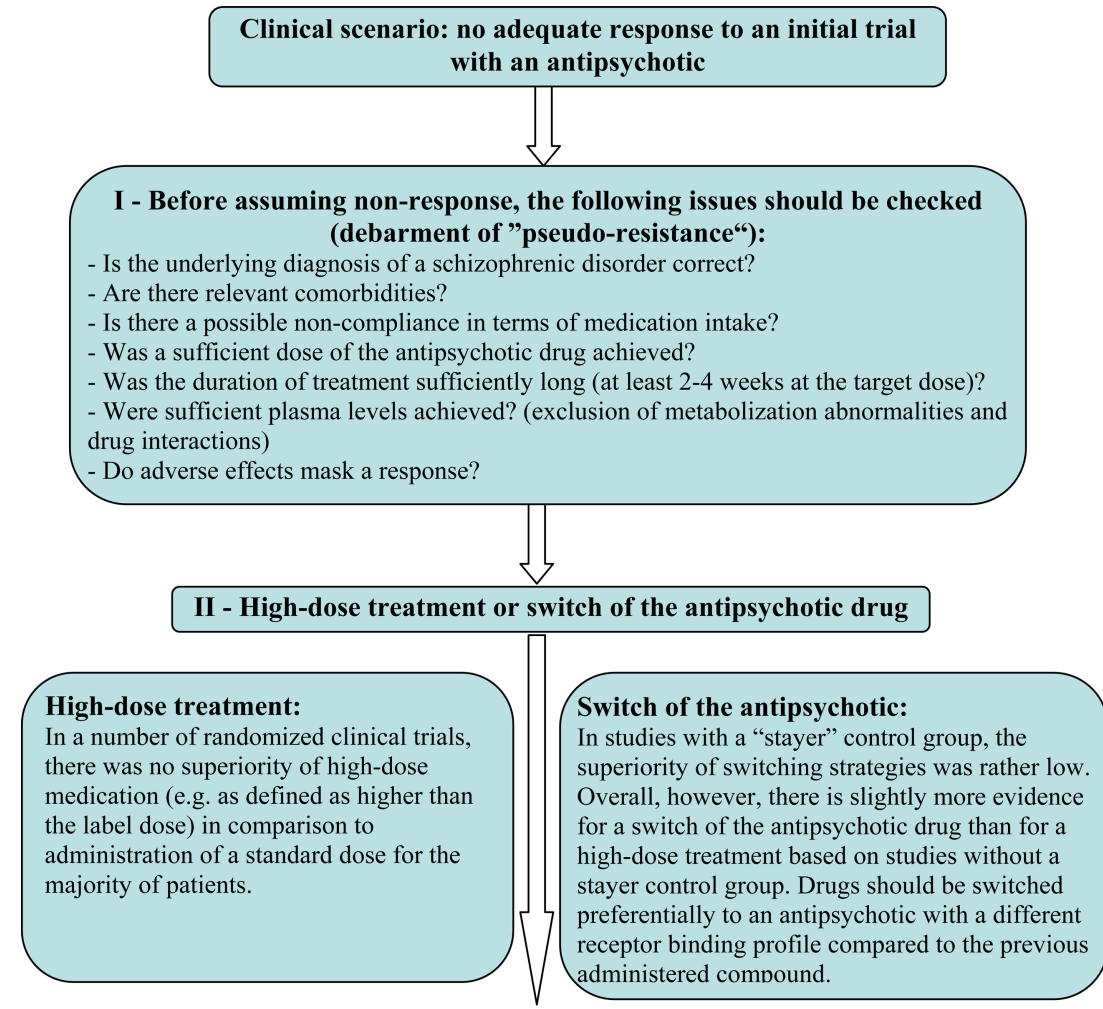

III - Medication with clozapine:

Should be considered after non-response to at least two trials with different antipsychotic agents. Minimum treatment duration: eight weeks, plasma-level guided.

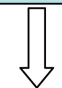

IV - combination and augmentation strategies:

- Currently, there is no sufficient convincing evidence to recommend such strategies generally. An antipsychotic monotherapy should be sought primarily.

- Utilization preferably for treating specific target symptoms (e.g. benzodiazepines

for agitated patients or antidepressants for affective symptoms).

- For combination treatment two antipsychotics with a different receptor binding profile should be chosen (e.g. potent D2-antagonists + multi-receptor-antagonists).

3. Was the antipsychotic dose sufficient and the treatment duration adequate? Recommended doses, based on an international consensus survey, for the most frequently prescribed antipsychotic drugs are displayed in table $1 .{ }^{13}$ Patients with first-episode schizophrenia as well as older patients often require lower doses. The onset of symptom improvement may vary considerably between individual patients. International pharmacological guidelines recommend a minimum medication period between 2 and 8 weeks until in case of insufficient response a change of the treatment strategy should be considered. ${ }^{1}{ }^{3} 4$ The well-established theory of the delayed onset of antipsychotic action followed by most of the guidelines has been increasingly discussed and critically questioned over the past years. Agid et $\mathrm{al}^{14}$ showed that the highest symptom reduction can be observed already within the first week of medication and then decreases consistently. All in all, the study results suggest that in case of no symptom improvement within the first 2 weeks of treatment in adequate dose, no long-term response to therapy can be expected. ${ }^{15}$ Some studies even postulate that a statistically significant antipsychotic effect can already be observed within the first $24 \mathrm{~h}$ after administration of the medication. ${ }^{16}$ Analyses of clinical trials in treatment-resistant schizophrenia could display that in patients with a history of at least one non-response to antipsychotic treatment a significant symptom reduction occurs within the first 4 weeks. ${ }^{17}$

4. Side effects of antipsychotics can mask treatment response. For example, akathisia can be misinterpreted as mental agitation or parkinsonism may mimic schizophrenic negative symptoms.

5. Were sufficient plasma levels achieved? Currently, there is no convincing evidence for a clear relationship between drug concentrations in the blood and antipsychotic response, and an exact dose titration guided by therapeutic drug monitoring (TDM) may be justified for clozapine, at best. ${ }^{13}$ However, plasma level measurements may be useful in clinical practice in case of inefficacy of the medication or occurrence of severe adverse effects even at low doses. Drug concentration in the blood should be determined in the steady-state which is attained for most of the psychopharmacological drugs after approximately five elimination half-lives after the first medication intake. This corresponds for most antipsychotics to 1 week after achievement of the maintenance dose. ${ }^{18}$ Blood for drug concentration measurement should be withdrawn ideally before ingestion of the morning dose which is usually $12-16 \mathrm{~h}$ (or $24 \mathrm{~h}$ if the drug is given once daily in the morning) after the last medication intake. ${ }^{18}$ Applying TDM, metabolisation abnormalities as well as insufficient compliance of the patient can be identified or excluded as reason 
Table 1 Recommended doses of selected first-generation and second-generation antipsychotic drugs for the pharmacological treatment of schizophrenia and related disorders based on an international consensus survey ${ }^{13}$

\begin{tabular}{lclc}
\hline Drug & $\begin{array}{l}\text { Recommended } \\
\text { starting dose } \\
\text { (in mg/day) }\end{array}$ & $\begin{array}{l}\text { Recommended } \\
\text { target dose } \\
\text { (in mg/day) }\end{array}$ & $\begin{array}{l}\text { Recommended } \\
\text { maximum dose } \\
\text { (in mg/day) }\end{array}$ \\
\hline First-generation antipsychotic drugs & & \\
Chlorpromazine & 100 & $300-600$ & 800 \\
$\quad$ Flupentixol & 3 & $5-12$ & 18 \\
Fluphenazine & 3 & $5-15$ & 20 \\
Haloperidol & 3 & $5-10$ & 20 \\
Perphenazine & 8 & $12-24$ & 42 \\
& 5 & $10-20$ & 35 \\
Trifluoperazine & & & \\
Second-generation antipsychotic drugs & & 1000 \\
Amisulpride & 100 & $400-800$ & 30 \\
Aripiprazole & 10 & $15-30$ & 800 \\
Clozapine & 25 & $200-500$ & 30 \\
Olanzapine & 5 & $10-20$ & 12 \\
Paliperidone & 3 & $6-9$ & 1000 \\
Ouetiapine & 100 & $400-800$ & 8,5 \\
Risperidone & 2 & $4-6$ & 200 \\
Ziprasidone & 40 & $120-160$ & 400 \\
Zotepine & 50 & $100-300$ & \\
\hline
\end{tabular}

Some groups of patients require normally lower doses for achieving response, for example patients with first-episode schizophrenia as well as older patients.

for treatment failure. Polymorphisms in the cytochrome P450 enzyme system, which is responsible for the metabolisation of most psychotropic drugs, can be also detected. On the one hand, increased enzyme activity can cause an accelerated metabolisation of the drugs ('ultrarapid metaboliser', about 1\% of the population), on the other hand, reduced enzyme activity can cause a slower metabolisation ('poor metaboliser', about 5\% of the population). The compounds can therefore accumulate in the body even at very low doses and in some cases toxic drug-concentration levels can be achieved. The occurrence of severe adverse effects even at low doses can be interpreted as a sign for possible pharmacokinetic problems. Moreover, potential drug interactions should be considered in case of pharmacological combination therapies. Clinically relevant in this context is the interaction between smoking and a medication with drugs that are metabolised mainly by the cytochrome P450 isoenzyme 1A2 such as the antipsychotics clozapine and olanzapine. As smoking induces this isoenzyme, the clearance of these compounds can be increased significantly. Therefore, a dose escalation may be necessary to achieve the therapeutic window (the blood concentration in which a drug is most effective and the occurrence of adverse effects is low).

\section{Dose increase or switching of the antipsychotic drug}

In case of non-response to an initial antipsychotic drug treatment, two strategies that are often used in clinical routine care are a dose increase of the current administered antipsychotic agent (dose escalation, highdose treatment) and a switch to another, new antipsychotic drug.

\section{Dose increase}

A high-dose treatment with an increase of the antipsychotic dose above the officially approved dose range (off-label dose, dose escalation) cannot be recommended as general treatment option for the management of treatment-resistant schizophrenia. This opinion is stated consistently in all treatment guidelines of the international psychiatric societies. ${ }^{1}{ }^{3}{ }^{4}$ With regard to first-generation antipsychotics many clinical studies and systematic reviews concluded that a daily dose more than 800-1000 mg chlorpromazine equivalents (or even lower) does not improve antipsychotic efficacy but is associated with an increased incidence of especially extrapyramidal adverse effects. ${ }^{19}$ Although dose escalation cannot be advised generally, individual patients may respond to a high-dose or even off-label treatment. Applying blood monitoring of drugs raises the possibility of identifying patients that could probably benefit from high-dose medication. ${ }^{18}$ Thus, for example, patients with polymorphisms in the cytochrome P450 enzyme system which cause an accelerated elimination of drugs ('rapid metaboliser') can probably benefit from a dose increase when their drug concentration in the blood is below the effective therapeutic range in a standard dose. When a high-dose therapy is initiated in a patient, it should be closely evaluated and in case of inefficacy, again a standard dose of the antipsychotic should be sought.

\section{Switching the antipsychotic drug}

Although a switch of the currently administered antipsychotic drug is an often employed step in case of non-response, the question of the effectiveness of switching from one antipsychotic drug to a second (each as monotherapy) is still open. Even though the efficacy of all officially approved antipsychotic drugs is verified in positive clinical trials it must be considered that these findings are derived from data with nonresistant patients and these results cannot be automatically extrapolated to participants who have no or inadequate response to previous antipsychotic medication. Ideally, to evaluate a switching strategy trials are needed in which non-responders to antipsychotic pharmacotherapy are randomised in at least two study arms: in the intervention groups the participants are switched to a new antipsychotic drug (switching group) and in the control group the medication is continued with the initial antipsychotic without any dose adjustments. We found a doubleblind, randomised study with such a design. ${ }^{20}$ The authors randomised non-responders to an initial 2-week monotherapy with risperidone to either a switch to olanzapine $(10-20 \mathrm{mg} /$ day, $n=186)$ or continuing the same dose of risperidone $(2-6 \mathrm{mg} /$ day, $\mathrm{n}=192)$ in the control group. After 10 weeks, a small but statistically significant between-group difference could be identified in favour of switching to olanzapine (mean difference on the 'Positive and Negative Syndrome Scale': 3.5 points). In an earlier randomised study by Kinon et $\mathrm{al}^{21}{ }^{21}$ non-responders to a 4-week monotherapy with fluphenazine $20 \mathrm{mg} /$ day $(\mathrm{n}=115)$ were randomly assigned to the following study groups: continuation of the medication with fluphenazine in a constant dose, dose-escalation of fluphenazine to $80 \mathrm{mg} /$ day or a switch to haloperidol $20 \mathrm{mg} /$ day. There was no significant superiority for one of the three study groups.

Additionally, randomised trials without an adequate control group with continuation of the initial antipsychotic monotherapy have been carried out. $^{22-26}$ However, due to this methodological limitation, their findings must be interpreted with caution. In conclusion, there is insufficient evidence for clear therapeutic recommendations with regard to a switching strategy. Even if only moderate-to-low response rates can be expected, some patients appear to benefit from a switch of medication. From an evidence-based perspective, we see slight advantages of this strategy compared to a high-dose treatment with antipsychotics. However, it must be considered that there are individual patients who may also benefit from a high-dose medication. In this regard, the medical history of the patient must be assessed and taken into account.

For switching the antipsychotic drug, it is recommended to taper off the dose of the first antipsychotic gradually while simultaneously the dose of the second one is titrated up gradually to its target dose ('crossover titration'). Alternatively, the dose of the first antipsychotic can be maintained at the same dose while the dose of the second 
compound is increased gradually to a therapeutic level and only then the dose of the first agent will be decreased ('overlap and taper'). ${ }^{1} 10$

Following pharmacodynamic considerations in case of switching, it seems preferable to choose a new compound with a different receptorbinding profile compared to the first administered agent leg, a drug with high serotonergic properties such as quetiapine, if the first compound was characterised by high affinities to dopamine receptors such as amisulpride, risperidone or a first generation antipsychotic agent). Similarly, the results of the phase $1 \mathrm{~b}$ study of the 'Clinical Antipsychotic Trials of Intervention Effectiveness (CATIE) ${ }^{\prime 27}$ can be interpreted in this sense. ${ }^{28}$ In this trial, non-responders to perphenazine benefited significantly more from a switch to olanzapine or quetiapine compared to risperidone; probably because both perphenazine and risperidone are characterised by high antidopaminergic properties and therefore, a switch to a compound with a different mechanism of action seems to be a more promising approach for perphenazine non-responders.

\section{Pharmacotherapy with clozapine}

A large number of clinical trials were conducted to elucidate which antipsychotic is characterised by the highest antipsychotic efficacy. The second-generation antipsychotics clozapine, amisulpride, olanzapine and risperidone appear to be slightly more effective in terms of antipsychotic efficacy than the other antipsychotic drugs. ${ }^{29} 30$ However, with the exception of clozapine the differences in effect sizes are small. In a network meta-analysis comprising 212 randomised trials and a total of 43049 patients with schizophrenia, clozapine achieved the highest effect size in terms of antipsychotic efficacy followed by amisulpride, olanzapine and risperidone. ${ }^{30}$ Interestingly, studies with therapy-resistant patients were excluded in this analysis and clozapine was superior even in non-resistant participants. However, the authors discussed that the superiority of clozapine stems mainly from comparisons with first-generation rather than second-generation antipsychotics.

Particularly, when considering the evidence for treatment-resistant patients, clozapine is characterised by high efficacy verified in a large number of randomised trials. Therefore, clozapine is currently classified as first-line treatment in treatment-resistant schizophrenia. ${ }^{13-5}$ This advice is corroborated by an early meta-analysis that investigated exclusively treatment-resistant participants and determined that only clozapine was significantly more efficacious than first-generation antipsychotics. ${ }^{31}$ Similarly, clozapine resulted superior to other second-generation antipsychotics in a phase II study of the CATIE trial ${ }^{32}$ and in the 'Cost Utility of the Latest Antipsychotic Drugs in Schizophrenia Study (CUtLASS 2)' when analysing schizophrenic symptom improvement. ${ }^{33}$

In conclusion, based on the current evidence clozapine remains the gold standard and mainstay in the pharmacological treatment of refractory schizophrenia. However, due to its risk profile (especially in terms of agranulocytosis, which is about $1 \%)$, clozapine may be used only after at least two failed previous treatment attempts with two other different antipsychotics in adequate dosage and duration. ${ }^{1}{ }^{3} 4$ Further important limitations of clozapine in comparison to other antipsychotics arise from the necessity of slow dose titration and weekly blood counts within the first 18 weeks of treatment (and subsequently every month). Especially when high clozapine doses are administered, the reduced threshold for seizures must be considered. In clinical practice, mainly sedation, weight gain and hypersalivation may hamper the compliance of patients.

\section{The role of other second-generation antipsychotics in treatment-resistant schizophrenia}

Although the efficacy of clozapine in the pharmacological management of treatment-resistant schizophrenia is undisputed and sufficiently proved, an ambiguity remains regarding the status of other antipsychotics in treatment-resistant conditions. Significant effect sizes were also found for the second-generation antipsychotics risperidone, olanzapine and amisulpride. ${ }^{29} 30$ However, these data were mainly derived from non-resistant participants. Uncertainty still exists which antipsychotic should be given when clozapine treatment is not tolerated or is not feasible because of the occurrence of adverse effects. In this case, some treatment guidelines recommend explicitly a treatment preferably with olanzapine or risperidone. ${ }^{1}$ In clinical trials with treatment-resistant patients, both compounds were not significantly inferior in direct comparison to clozapine and were able to achieve higher effect sizes compared to first-generation antipsychotics. ${ }^{10}$

\section{Combination strategies}

Pharmacological combination treatment is defined as the simultaneous administration of two drugs of the same group such as two antipsychotics. Although this strategy is widely used in daily clinical practice, ${ }^{34}$ the results of meta-analyses and systematic reviews are rather sobering. In their meta-analysis comprising 14 double-blind, randomised, placebo-controlled clozapine combination studies with secondgeneration antipsychotics, Taylor et $a^{35}$ found a small but statistically significant superiority of the combination treatment compared to placebo. In another meta-analysis by Sommer et al, ${ }^{36}$ stratified according to the various compounds combined with clozapine, a significant positive effect was determined only for sulpiride (based on a single trial) but not for amisulpride, aripiprazole, risperidone and haloperidol. Barbui et $a \beta^{37}$ found a significant superiority of clozapine combination with second-generation drugs only for randomised open studies but not for double-blind trials. Correll et a ${ }^{38}$ compared in their meta-analysis an antipsychotic combination with monotherapy whereas the previously described meta-analyses exclusively examined clozapine cotreatment. The combination therapy was superior to monotherapy in terms of allcause discontinuation and dropouts due to inefficacy. However, it must be noted that many Chinese studies were included that did not comprise only treatment-resistant participants. Furthermore, in this meta-analysis a publication bias cannot be ruled out. ${ }^{38}$

Currently, clozapine is the most evaluated antipsychotic drug regarding combination treatments. The most frequently investigated single combination is that of clozapine with risperidone. From a pharmacological point of view, it seems auspicious to combine antipsychotic agents with low antidopaminergic properties such as clozapine with antipsychotics that are characterised by a particularly strong affinity to dopamine receptors, such as amisulpride, sulpiride, haloperidol or risperidone. Concerning the partial dopamine agonist aripiprazole, there is no convincing evidence that a combination treatment with this compound causes any improvement in schizophrenic symptoms ${ }^{36}{ }^{39}$ but it seems that cotreatment with aripiprazole can reduce antipsychotic-induced metabolic adverse effects as well as elevated serum prolactin levels. ${ }^{39}$ Antipsychotic monotherapy should be generally preferred and sought, although individual patients can certainly benefit from a combination treatment. In this case, efficacy, drug interactions and the occurrence of adverse effects should be closely monitored and, in case of inefficacy of the combination strategy, monotherapy should be considered again. In particular, the risk of metabolic side effects and all-cause discontinuation may increase significantly by administering antipsychotic combinations. ${ }^{40}$ In a retrospective Hungarian cohort study, a switch of an antipsychotic drug $(n=5480)$ was compared to combination treatment with two antipsychotics $(n=7901)$ in patients with at least one previous drug treatment failure. Premature discontinuation occurred significantly earlier in the combination treatment group than in the switching group. Otherwise, in terms of mortality and hospitalisation rates, the pharmacological combination therapy was superior to monotherapy. ${ }^{41}$

\section{Augmentation strategies}

Augmentation treatment means the concomitant use of two drugs of different classes, for example the coadministration of an antipsychotic 
drug with an antidepressant, mood stabiliser or benzodiazepine. Many compounds have been investigated as augmentor of antipsychotics without demonstrating convincing efficacy in treating schizophrenic symptoms. Evaluated agents are, for example, acetylcholinesterase inhibitors, $\beta$-blockers, carbamazepine, lithium, valproate and memantine. ${ }^{10}$ Although benzodiazepines may be indicated in short-term treatment of acutely agitated patients, there is no evidence for the use of benzodiazepines as long-term adjunctive treatment to improve psychotic symptoms. $^{42}$ Sommer et a/ ${ }^{36}$ found in their meta-analysis a significant positive effect of lamotrigine augmentation in clozapine-resistant schizophrenia but this effect disappeared in a sensitivity analysis after exclusion of an outlier study with high effect size and small sample size. Similarly, a significant positive effect of topiramate on schizophrenic positive symptoms diminished after removal of an outlier study. ${ }^{36}$ Very recent meta-analyses support augmentation with aspirin or other drugs with effects on the immune system, but these findings are in our opinion not yet ready for transfer into practice. ${ }^{43} 44$

As with the combination treatments, there is no sufficient evidence to advise the general use of pharmacological augmentation strategies in treatment-resistant schizophrenia. A possible increase of adverse effects and drug interactions must be considered. Augmentation strategies should be regarded preferable for the treatment of specific target symptoms such as benzodiazepines in highly agitated psychotic patients ${ }^{45}$ or antidepressants for the treatment of comorbid depressive symptoms or marked negative symptoms. ${ }^{46}$ Augmentation and combination treatments should be discontinued in case of inefficacy and antipsychotic monotherapy should be sought again.

\section{Competing interests None.}

doi:10.1136/eb-2014-101813

\section{REFERENCES}

1. Hasan A, Falkai P, Wobrock T, et al. World Federation of Societies of Biological Psychiatry (WFSBP) Guidelines for Biological Treatment of Schizophrenia, part 1 update 2012 on the acute treatment of schizophrenia and the management of treatment resistance. World J Biol Psychiatry 2012:13:318-78.

2. Suzuki T, Remington G, Mulsant BH, et al. Treatment resistant schizophrenia and response to antipsychotics: a review. Schizophr Res 2011;133:54-62.

3. Buchanan RW, Kreyenbuhl J, Kelly DL, et al. The 2009 schizophrenia PORT psychopharmacological treatment recommendations and summary statements. Schizophr Bull 2010;36:71-93.

4. Lehman AF, Lieberman JA, Dixon LB, et al. Practice guideline for the treatment of patients with schizophrenia, second edition. Am J Psychiatry 2004;161/Suppl 2):1-56.

5. NICE. Schizophrenia: full national guideline on core interventions in primary and secondary care. National Institute for Clinical Excellence, 2003.

6. Kane J, Honigfeld G, Singer J, et al. Clozapine for the treatment-resistant schizophrenic. A double-blind comparison with chlorpromazine. Arch Gen Psychiatry 1988;45:789-96.

7. Suzuki T, Remington G, Mulsant BH, et al. Defining treatment-resistant schizophrenia and response to antipsychotics: a review and recommendation. Psychiatry Res 2012;197:1-6.

8. Demjaha A, Murray RM, McGuire PK, et al. Dopamine synthesis capacity in patients with treatment-resistant schizophrenia. Am J Psychiatry 2012;169:1203-10.

9. Dold M, Leucht S. Pharmakotherapie der therapieresistenten Schizophrenie. PSYCH up2date, 2014.

10. Leucht S, Heres S, Kissling W, et al. Pharmacological treatment of schizophrenia Fortschr Neurol Psychiatr 2013;81:e1-13.

11. Leucht S, Heres S, Kissling W, et al. Evidence-based pharmacotherapy of schizophrenia. Int J Neuropsychopharmacol 2011;14:269-84.

12. Goff DC, Hill M, Freudenreich 0 . Strategies for improving treatment adherence in schizophrenia and schizoaffective disorder. J Clin Psychiatry 2010;71(Suppl 2):20-6.

13. Gardner DM, Murphy AL, O'Donnell H, et al. International consensus study of antipsychotic dosing. Am J Psychiatry 2010;167:686-93.

14. Agid 0, Kapur S, Arenovich T, et al. Delayed-onset hypothesis of antipsychotic action: a hypothesis tested and rejected. Arch Gen Psychiatry 2003;60:1228-35.

15. Leucht S, Busch R, Kissling W, et al. Early prediction of antipsychotic nonresponse among patients with schizophrenia. J Clin Psychiatry 2007:68:352-60.

16. Kapur S, Arenovich T, Agid 0, et al. Evidence for onset of antipsychotic effects within the first 24 hours of treatment. Am J Psychiatry 2005;162:939-46.
17. Suzuki T, Remington G, Arenovich T, et al. Time course of improvement with antipsychotic medication in treatment-resistant schizophrenia. Br J Pychiatry 2011;199:275-80.

18. Hiemke C, Baumann P, Bergemann N, et al. AGNP consensus guidelines for therapeutic drug monitoring in psychiatry: update 2011. Pharmacopsychiatry 2011;44:195-235.

19. Davis JM, Chen N. Dose response and dose equivalence of antipsychotics. J Clin Psychopharmacol 2004;24:192-208

20. Kinon BJ, Volavka J, Stauffer V, et al. Standard and higher dose of olanzapine in patients with schizophrenia or schizoaffective disorder: a randomized, double-blind fixed-dose study. J Clin Psychopharmacol 2008;28:392-400.

21. Kinon BJ, Kane JM, Johns C, et al. Treatment of neuroleptic-resistant schizophrenic relapse. Psychopharmacol Bull 1993;29:309-14

22. Takahashi H, Kamata M, Yoshida K, et al. Switching to olanzapine after unsuccessful treatment with risperidone during the first episode of schizophrenia: an open-label trial. J Clin Psychiatry 2006;67:1577-82.

23. Takahashi H, Yoshida K, Ishigooka J, et al. Switching to risperidone after unsuccessful treatment of olanzapine in the first-episode schizophrenia: an open trial. Prog Neuropsychopharmacol Biol Psychiatry 2006;30:1067-72.

24. Agid 0, Schulze L, Arenovich T, et al. Antipsychotic response in first-episode schizophrenia: efficacy of high doses and switching. Eur Neuropsychopharmacol 2013;23:1017-22.

25. Shalev A, Hermesh $\mathrm{H}$, Rothberg J, et al. Poor neuroleptic response in acutely exacerbated schizophrenic patients. Acta Psychiatr Scand 1993;87:86-91.

26. Suzuki T, Uchida $\mathrm{H}$, Watanabe $\mathrm{K}$, et al. How effective is it to sequentially switch among olanzapine, quetiapine and risperidone? A randomized, open-label study of algorithm-based antipsychotic treatment to patients with symptomatic schizophrenia in the real-world clinical setting. Psychopharmacol 2007;195:285-95.

27. Lieberman JA, Stroup TS, McEvoy JP, et al. Effectiveness of antipsychotic drugs in patients with chronic schizophrenia. N Engl J Med 2005;353:1209-23.

28. Stroup TS, Lieberman JA, McEvoy JP, et al. Effectiveness of olanzapine, quetiapine, and risperidone in patients with chronic schizophrenia after discontinuing perphenazine: a CATIE study. Am J Psychiatry 2007;164:415-27.

29. Leucht S, Corves C, Arbter D, et al. Second-generation versus first-generation antipsychotic drugs for schizophrenia: a meta-analysis. Lancet 2009;373:31-41.

30. Leucht S, Cipriani A, Spineli L, et al. Comparative efficacy and tolerability of 15 antipsychotic drugs in schizophrenia: a multiple-treatments meta-analysis. Lancet 2013:382:951-62.

31. Chakos M, Lieberman J, Hoffman $\mathbf{E}$, et al. Effectiveness of second-generation antipsychotics in patients with treatment-resistant schizophrenia: a review and meta-analysis of randomized trials. Am J Psychiatry 2001;158:518-26.

32. McEvoy JP, Lieberman JA, Stroup TS, et al. Effectiveness of clozapine versus olanzapine, quetiapine, and risperidone in patients with chronic schizophrenia who did not respond to prior atypical antipsychotic treatment. Am J Psychiatry 2006;163:600-10.

33. Lewis SW, Barnes TR, Davies L, et al. Randomized controlled trial of effect of prescription of clozapine versus other second-generation antipsychotic drugs in resistant schizophrenia. Schizophr Bull 2006;32:715-23.

34. Gallego JA, Bonetti J, Zhang J, et al. Prevalence and correlates of antipsychotic polypharmacy: a systematic review and meta-regression of global and regional trends from the 1970s to 2009. Schizophr Res 2012;138:18-28.

35. Taylor DM, Smith L, Gee SH, et al. Augmentation of clozapine with a second antipsychotic - a meta-analysis. Acta Psychiatr Scand 2012;125:15-24.

36. Sommer IE, Begemann MJ, Temmerman A, et al. Pharmacological augmentation strategies for schizophrenia patients with insufficient response to clozapine: a quantitative literature review. Schizophr Bull 2012;38:1003-11.

37. Barbui C. Signoretti A, Mule $S$, et al. Does the addition of a second antipsychotic drug improve clozapine treatment? Schizophr Bull 2009;35:458-68.

38. Correll CU, Rummel-Kluge C, Corves C, et al. Antipsychotic combinations vs monotherapy in schizophrenia: a meta-analysis of randomized controlled trials Schizophr Bull 2009:35:443-57.

39. Fleischhacker WW, Uchida H. Critical review of antipsychotic polypharmacy in the treatment of schizophrenia. Int J Neuropsychopharmacol 2012:1-11.

40. Gallego JA, Nielsen J, De Hert M, et al. Safety and tolerability of antipsychotic polypharmacy. Expert Opin Drug Saf 2012;11:527-42.

41. Katona L, Czobor P, Bitter I. Real-world effectiveness of antipsychotic monotherapy vs. polypharmacy in schizophrenia: To switch or to combine? A nationwide study in Hungary. Schizophr Res 2014;152:246-54.

42. Dold M, Li C, Gillies D, et al. Benzodiazepine augmentation of antipsychotic drugs in schizophrenia: a meta-analysis and Cochrane review of randomized controlled trials. Eur Neuropsychopharmacol 2013;23:1023-33.

43. Sommer IE, van Westrhenen R, Begemann MJ, et al. Efficacy of anti-inflammatory agents to improve symptoms in patients with schizophrenia: an update. Schizophr Bull 2014;40:181-91.

44. Sommer IE, de Witte L, Begemann M, et al. Nonsteroidal anti-inflammatory drugs in schizophrenia: ready for practice or a good start? A meta-analysis. J Clin Psychiatry 2012:73:414-19.

45. Dold M, Li C, Tardy M, et al. Benzodiazepines for schizophrenia. Cochrane Database Syst Rev 2012;11:CD006391.

46. Singh SP, Singh V, Kar N, et al. Efficacy of antidepressants in treating the negative symptoms of chronic schizophrenia: meta-analysis. Br J Psychiatry 2010;197:174-9. 